Review

\title{
Emerging Roles of SIRT1 in Alcoholic Liver Disease
}

\author{
Ruixue Ren ${ }^{1}$, Ziming Wang1, Miaomiao $\mathrm{Wu}^{2,3}$, Hua Wang1,2,3 \\ 1. Department of Oncology, the First Affiliated Hospital of Anhui Medical University, Hefei 230022, Anhui, China \\ 2. School of Pharmacy, Institute of Liver Diseases, Anhui Medical University, Hefei 230032, Anhui, China \\ 3. Inflammation and Immune Mediated Diseases Laboratory of Anhui Province, Hefei 230032, Anhui, China
}

$\triangle$ Corresponding author: Dr. Hua Wang, Department of Oncology, the First Affiliated Hospital of Anhui Medical University; 218 Jixi Road, Hefei 230022, Anhui, China. Email: wanghua@ahmu.edu.cn

(c) The author(s). This is an open access article distributed under the terms of the Creative Commons Attribution License (https://creativecommons.org/licenses/by/4.0/). See http://ivyspring.com/terms for full terms and conditions.

Received: 2020.06.15; Accepted: 2020.09.21; Published: 2020.10.17

\begin{abstract}
Alcoholic liver disease (ALD) is the most prevalent type of chronic liver disease worldwide with a wide spectrum of liver pathologies ranging from simple steatosis to steatohepatitis, cirrhosis, and even hepatocellular carcinoma. It has been demonstrated that ALD is mediated in whole or in part by a central signaling molecule sirtuin 1 (SIRT1), a conserved class III histone deacetylase.SIRT1 plays beneficial roles in regulating hepatic lipid metabolism, inhibiting hepatic inflammation, controlling hepatic fibrosis and mediating hepatocellular carcinoma in ALD. However, underlying molecular mechanisms are complex and remain incompletely understood. The aim of this review was to highlight the latest advances in understanding of SIRT1 regulatory mechanisms in ALD and discuss their unique potential role as novel therapeutic target for ALD treatment.
\end{abstract}

Key words: Alcoholic liver disease; SIRT1; Inflammation; Fibrosis; Hepatocellular carcinoma

\section{Introduction}

Alcohol abuse is the leading cause of alcoholic liver disease (ALD) worldwide [1]. Now, ALD has becoming a major public health problem worldwide and a leading cause of morbidity and mortality worldwide. It covers multiple pathological types, including simple steatosis, alcoholic steatohepatitis (ASH), progressive fibrosis, advanced cirrhosis, and hepatocellular cancer (HCC) [2,3]. Notably, ASH is recognized as a potential precursor for fibrosis and hepatocellular carcinoma [2, 4].Despite extensive research on understanding the mechanism of ALD, there are still no targeted therapies available.

The sirtuins are a family of evolutionarily conserved NAD-dependent class III histone deacetylase [5-7]. The sirtuins family consist of seven members (SIRT1 - 7) and sirtuin 1 (SIRT1) is the most extensively studied among them [8]. They regulate many cellular and physiological processes in normal and diseased conditions [7]. Recent reports indicate that sirtuins play important roles in regulating the ALD related metabolic processes [9-11]. Early studies reported that ethanol exposure reduces SIRT1 gene and protein expression levels[11].The decreased
SIRT1 levels plays crucial roles in the regulation of ALD by modifying the acetylation status of various target molecules, including histones, transcriptional regulators, and its co-regulators [11-14]. In this review, we will summarize the latest advances about roles of SIRT1 in ALD, with a focus on how SIRT1 regulates lipid metabolism, oxidative stress and inflammation, fibrosis and HCC in the liver. We will also discuss the more potential mechanisms of alcohol regulation of SIRT1 levels in ALD and applications of SIRT1 activators as therapeutic agents for ALD treatment.

\section{Ethanol regulates hepatic SIRT1}

As summarized in figure 1, ethanol exposure decreases SIRT1 expression levels, and ultimately inhibits SIRT1 deacetylase activity in the liver [11, 15-17]. However, the specific mechanism needs further clarification.

Ethanolis mainly metabolized through the oxidative pathways that are mediated by alcohol dehydrogenase $(\mathrm{ADH})$ and aldehyde dehydrogenase $(\mathrm{ALDH})$, which resulting in reduction of $\mathrm{NAD}^{+}[2]$. 
SIRT1 is a NAD(+)-dependent deacetylase and thus ethanol mediated decreased $\mathrm{NAD}^{+} / \mathrm{NADH}$ levels could inhibit SIRT1 activity in liver [18, 19]. SIRT1 is highly sensitive to intracellular oxidation state. In addition to the main oxidative metabolism pathways mentioned above, both the reaction catalyzed by microsomal CYPs, mainly CYP2E1, and the pathway mediated by peroxisomal CAT, compose a small percentage of alcohol oxidation[20, 21].The unfavorable products of oxidative metabolism in ALD, including acetaldehyde and acetate, may ultimately down-regulate hepatic SIRT1 and activity [22]. Interestingly, deletion of SIRT1 drastically exacerbates ethanol mediated oxidative stress, indicating that the inhibition of SIRT1 in ALD may format a feedback loop to further suppress SIRT1 through ROS production [15]. In addition, the localization of SIRT1 determines its activity and function. In general, SIRT1 is predominately located in the nucleus and exert protective roles in liver, as reported previously [23]. SIRT1 translocated from the nucleus to the cytoplasm from the nucleus to the cytoplasm when liver tissue exposure to alcohol [24]. Therefore, ethanol exposure increase ROS production, decrease $\mathrm{NAD}^{+} / \mathrm{NAD}$ ratio and disturb SIRT1 nucleocytoplasmic shuttling, and ultimately inhibits SIRT1 deacetylase activity in the liver[25, 26].

In addition, microRNAs (miRs) have been recently shown to be widely involved in the development of ALD. MiR-217 is dramatically up-regulated in livers of chronically ethanol-fed mice and overexpression of miR-217 weaken ethanol-induced functions of SIRT1 [17, 27]. Therefore, hepatic miR-217 inhibition could be an attractive therapeutic approach for treating human alcoholic fatty liver disease. MiR-34a, another important microRNA in ALD progression, was also increased in ethanol-exposed mouse liver in vivo [28]. Up-regulated miR-34a level contributes to alcoholic liver injury through inhibiting SIRT1 expression [28]. Beyond miR-217 and miR-34a, other miRNAs are also known to regulate SIRT1 [29]. MiR-128-3p, miR-miR-9, MicroRNA-29a and miR-22all can reducedSIRT1 expression and exacerbated the inflammatory response [30-33]. However; their roles in the development of ALD will need to be further investigated.

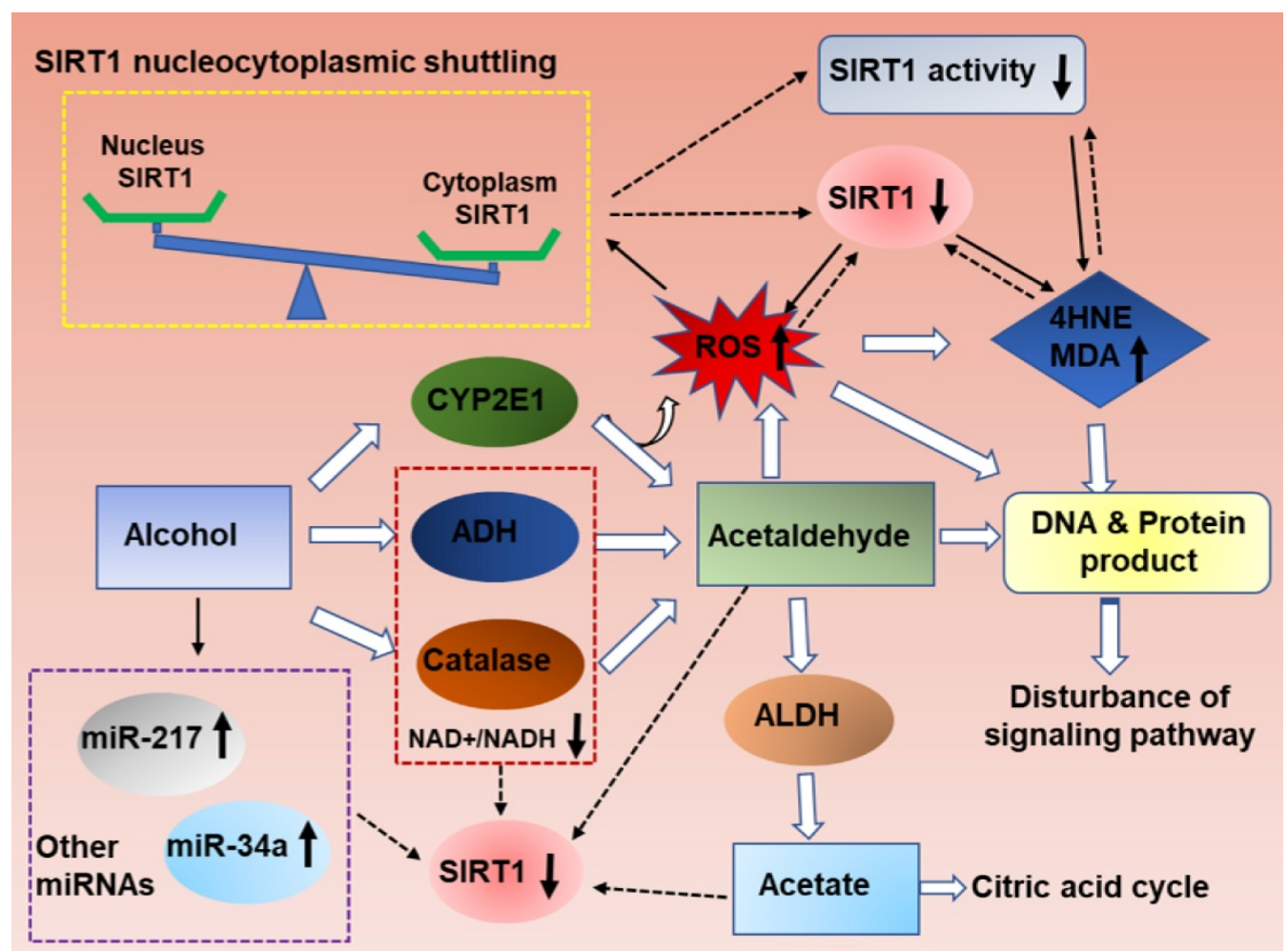

Figure 1. Mechanisms of hepatic SIRT1 expression and activity in response to ethanol challenge. Ethanol metabolism is a ROS generation process, which are carried out mainly by the enzymes alcohol dehydrogenase (ADH) and aldehyde dehydrogenase (ALDH), and partially by CYP2E1.Ethanol-induced oxidative stress directly down-regulate $\mathrm{NAD}^{+}$levels, increase ethanol metabolism product (acetaldehyde and acetate), increase SIRT1 nucleocytoplasmic shuttling, improve miRNAs that are associated with ALD progression, and ultimately inhibits SIRT1 gene and protein expression levels in the liver. The decreased SIRT1 levels in ALD result in liver injury and inflammation. Meanwhile, high levels excessive ethanol consumption can also directly damage cellular proteins and DNA and disturb ROS signaling, and then damage the liver tissue. 


\section{SIRT1 and steatosis/inflammation}

\subsection{SIRTI and steatosis}

Steatosis is characterized by the accumulation of triglycerides (TAGs) in the cytoplasm of hepatocytes. Recent studies indicate that ethanol mediated SIRT1 downregulation promote two key events in the development of steatosis. These events include alcohol stimulating lipogenesis and inhibiting fatty acid oxidation [11, 15, 34-36]. However, the underlying mechanism needs to be fully elucidated. Emerging evidence demonstrates that ethanol induced SIRT1 inhibition is mainly through disruption of a signaling network mediated by various transcriptional regulators and co-regulators, including termed mTOR complex 1 (mTORC1), sterol regulatory element-binding protein 1c (SREBP-1c), peroxisome proliferator-activated receptor a (PPARa), lipin-1, AMP-activated kinase (AMPK), adiponectin, nuclear transcription factor- $\mathrm{B}$ (NF-kB), PPAR $y$ co-activator-1a (PGC-1a)[11, 14, 37]. Disruption of the signaling network by ethanol via SIRT1 inhibition ultimately leads to steatosis and inflammatory injury in liver (Figure 2).

\section{SIRT1 andSREBP-1C}

Roles of SREBP-1c have been established as lipid synthetic transcription factors especially for cholesterol and fatty acid synthesis [38]. Previous studies have shown that ethanol causes liver steatosis by elevating SREBP-1cexpression, which gradually promote fatty acid synthesis in animal models [39]. Meanwhile, SIRT1 can directly interact with and deacetylates SREBP-1c and eventually inhibits SREBP-1C activity in regulation of hepatic lipid metabolism [40]. Therefore, inhibition of SIRT1 activity by ethanol feeding was related to an increase in the acetylated form of SREBP-1c, and consequently leading to the development of steatosis [26]. Therefore, regulation of SIRT1-SREBP-1c axis has been proposed as one of the underlying mechanisms linking ethanol exposure with hepatic steatosis development. Interestingly, alcohol also down-regulates factors that reduce SREBP-1c expression, such as AMP-activated protein kinase (AMPK), suggesting the regulation of SREBP-1 activity by SIRT1 via AMPK dependent- or independent-mechanisms in ALD [41].

\section{SIRT1 and DEPTOR-mTORC1}

The mechanistic target of rapamycin (mTOR), an evolutionarily conserved protein kinase, is part of the phosphoinositide 3-kinase (PI3K)-related family [42]. mTOR consist of two different functional complexes, known as mTOR complex 1 (mTORC1) and mTOR complex 2 (mTORC2)[42, 43]. DEP domain-containing mTOR-interacting protein (DEPTOR) has recently been proved an mTOR binding protein that inhibits the mTOR kinase [44, 45]. The present study demonstrates thatmTORC1 activation plays a causal role in alcoholic steatosis, inflammation, and liver injury. Hepatocyte-specific deletion of SIRT1 disrupts DEPTOR signaling pathway, promotes mTORC1 activation, and exacerbates the development of alcoholic fatty liver and liver injury in mice [46]. Mechanically, Chronic alcohol consumption causes SIRT1 suppression in hepatocytes, which lead to the downregulation of DEPTOR and activation of mTORC1 [46]. Further, studies show that aberrant activation of mTORC1 by alcohol stimulates transcriptional activity of SREBP-1, promotes the cytoplasmic translocation of lipin 1, and inhibits the transcriptional activity of PPARa, which in turn increases fatty acid synthesis and downregulates fatty acid oxidation [46].

\section{SIRTI and PPAR- $\alpha /$ PGC-1 $\alpha$}

PPAR- $\alpha$ and PGC-1a are prominent transcriptional regulators of lipid metabolism [47-49]. On one hand, studies found that ethanol consumption can indirectly inhibit PPAR-a via up-regulation of CYP2E1-derived oxidative stress [50]. On the other hand, SIRT1 regulates lipid homeostasis by positively regulating PPAR- $\alpha$ [12]. Therefore, SIRT1 downregulation in ethanol feeding mice reduce PPAR-a level, eventually leading to increased fatty acid synthesis. Of course, ethanol-induced damage of hepatic fatty acid oxidation and lipid accumulation, largely by inactivating hepatic PGC-1a, a key transcriptional coactivator for PPARa signaling pathway. SIRT1 directly interacts with, and deacetylates PGC-1a, which subsequently modulates PGC-1a activity [51, 52]. Hepatocyte-specific deletion of SIRT1 disturbs PGC-1a-PPARa signaling pathway, reduces fatty acid oxidation, and causes aggravated liver steatosis and inflammation [53]. Impairments of both PGC-1a and PPAR-a have been implicated in the development of alcoholic steatosis in animals. Therefore, it is likely that disruption of SIRT1-PGC-1a/PPAR-a axis by ethanol may act as one of the main triggers of ALD.

\section{SIRTI and AMPK}

The MAPK signaling pathway has been proved to play a key role in many biological processes, including cell growth, differentiation, metabolism, and response to environmental stress [54]. Recent studies demonstrated that AMPK/SIRT1 activation plays an important protective role in ethanolmediated liver diseases. Mechanically, AMPK can 
activate SIRT1 by increasing the substrate for SIRT1 activity that is NAD+ levels, and SIRT1 can also activate AMPK via the regulation of classical upstream AMPK kinase, liver kinase B1 [55]. Therefore, dysregulation of hepatic AMPK signaling pathway in response to chronic ethanol exposure act as a crucial mechanism for development of ALD in animals [56]. Meanwhile, some studies have also indicated that chronic ethanol exposure inhibited AMPK activity in cultured rat hepatocytes [56]. Ethanol-mediated inhibition of hepatic AMPK activity contributes to steatosis [41, 57, 58]. More fundamentally, ethanol-mediated impairment of SIRT1 and AMPK has been reported to be responsible for the reduction in PGC-1a and the increase in SREBP-1 activities in the livers of several alcohol-fed animal models [26, 41, 59]. Further research shows that resveratrol activates AMPK is SIRT1 dependent [60]. Resveratrol treatment improved SIRT1 activity and expression levels, which further stimulates AMPK activity in livers of ethanol-fed mice [35, 61]. Thus, the protective effect of resveratrol is partly dependent on the activation of SIRT1-AMPK signaling pathway in the livers of ethanol-fed mice [35].

\section{SIRT1 and lipin-1}

Lipin-1 is a protein that exhibits dual functions as a phosphatidic acid phosphohydrolase (PAP) enzyme in the TAGs synthetic pathways and a transcriptional co-regulator [62]. Previous studies have shown that ethanol exposure up-regulates the lipin-1 expression and promote production of cytosolic lipin-1 protein, resulting in increased PAP activity and TAGs synthesis in cultured hepatocytes and in mouse livers[63].Meanwhile, drastically decreased Lipin-1expression in the nucleus impaired nuclear lipin-1-mediated PGC-1a/PPARa transcriptional activity, two key mitochondrial genes involved in fatty acid oxidation [17, 63, 64]. Downregulation of nuclear-localized lipin-1promote activation of SREBP activity, leading to enhanced hepatic lipogenesis [65, 66]. In conclusion, ethanol-induced significant down-regulation of hepatic nucleus lipin-1 gene expression contributes to the abnormalities in hepatic lipid metabolism, ultimately leading to development of liver steatosis $[15,63]$.

SIRT1 appears to be the most upstream signaling pathway molecule targeted by ethanol, and lipin- 1 is a vital downstream regulator that may be responsible for ethanol-mediated signaling pathway interference that are controlled by SIRT1 in liver $[15,63]$. In the present, studies demonstrated that ethanol administration to Sirt1LKO mice disrupted lipin-1 signaling pathway, eventually resulting in steatosis and inflammation in the liver [15]. Mechanically, studies found that ethanol-mediated inhibition of SIRT1 leads to reduced serine/arginine rich splicing factor 10 (SFRS10) gene and protein expression levels in hepatocytes. In short, dysfunction of lipin-1 in ethanol metabolism is largely via SIRT1-SFRS10 inhibition [15]. We have already discussed that SIRT1 inhibition may directly perturb PGC-1a/PPAR, leading to decreased activities of fatty acid oxidation enzymes [2,53]. Interestingly, nuclear lipin-1 function is also a transcriptional co-activator by interacting with PGC-1a/PPAR-a[64]. The impaired function of the SIRT1-lipin-1 axis in alcoholic steatosis may lead to inhibited PGC-1a/PPAR-a [15, 64]. These findings clearly suggest the role of SIRT1-SFRS10-lipin-1 pathway in the development of alcoholic steatosis in mice.

\section{SIRT1 and Adiponectin}

Adiponectin is an adipocyte-derived cytokine and it has also been discussed that it protects the liver from alcohol-mediated damage [67, 68]. The adiponectin receptors (AdipoRs), including AdipoR1 and AdipoR2, mediate the metabolic actions of adiponectin [69]. Studies indicated that the development of alcoholic fatty liver is associated with reduced adiponectin levels, down-regulated hepatic adiponectin receptors, and disordered hepatic adiponectin signaling pathway in animals [70, 71]. Studies have shown that adiponectin exerts its protective roles in ALD mainly mediated by various transcriptional regulators, including SIRT1, AMPK, SREBP-1, Forkhead box O1 (FoxO1)and PGC-1a/PPAR-a, and eventually leading to enhanced lipid oxidation, reduced lipid synthesis and inhibition of hepatic steatosis [56, 71]. The down-regulated SIRT1 levels in chronically ethanol-fed mice can inhibit hepatic AdipoR1/R2 expression [71]. In turn, impairment of SIRT1 signaling pathway by ethanol exposure is, in whole or in part, due to ethanol-mediated inhibition of adiponectin in liver [72]. These results demonstrated thatSIRT1 is an important promotor in the events of adiponectin decreasing the hepatic steatosis in ethanol -fed mice. In addition, reductions in AMPK protein and PPAR-a DNA-binding activity in alcohol-fed animal were observed and treatment with adiponectin restored the ethanol inhibited PGC-1a/PPAR-a activity [68, 71]. These observations suggest that hepatoprotective roles of adiponectin in ALD are dependent on AMPK. Given that SIRT1-AMPK signaling pathway is upstream of SREBP-1c, stimulation of SIRT1-AMPK signaling pathway by adiponectin should also blunt ethanol-mediated SREBP-1c activation. However, it is 
still not clear whether or not adiponectin can directly block the ethanol stimulated SREBP-1c activation.

FoxO1, a major target of SIRT1, has been established as a key player in the regulation of lipid metabolism [73]. SIRT1-FoxO1 signaling pathway has been shown to affect hepatic AdipoR2 gene expression accompanied by improved hepatic FoxO1 hyperacetylation and enhanced nuclear FoxO1 protein levels in the livers of ethanol-fed mice [24].

\subsection{The role of SIRT 1 in ethanol-induced hepatic inflammation}

A growing body of evidences suggests that inflammatory processes are primary contributors to the development and progression of ALD, which are characterized by presence of inflammatory cells infiltration and high levels of pro-inflammatory cytokines [37, 74]. Many cell types in the liver involved in inflammation of ALD, including hepatocyte and myeloid cells, as well as other cell types [75]. NF-kB is a transcription factor which plays an important role in regulating inflammation [76]. It has been shown that SIRT1 participate in the pathogenesis of inflammation associated ethanol fatty liver diseases[22, 36, 37].Further mechanistic studies revealed that inhibition of SIRT1was associated with a marked increase in the acetylation of the RelA/p65 subunit of NF-kB and promotion of NF-KB transcriptional activity [22, 77]. Specifically, liver-specific deletion of SIRT1 exacerbates chronic-binge ethanol-induced fatty liver injury in mice, as indicated by substantially enhanced the levels of F4/80+ staining in the livers of ethanol-fed Sirt1LKO mice and elevated mRNA expression levels of pro-inflammatory cytokines compared with the livers of ethanol-fed WT mice[15]. Consistently, myeloid cell-specific disruption of SIRT1 in mice reveals that SIRT1 deficiency in macrophages induces NF-kB hyperacetylation, resulting in hepatic inflammation [78]. Conversely, mice with moderate overexpression of SIRT1 gene show beneficial effects on the liver by decreasing the proinflammatory cytokines including IL-6 and TNF-at through downregulation of NF-kB activity [79].

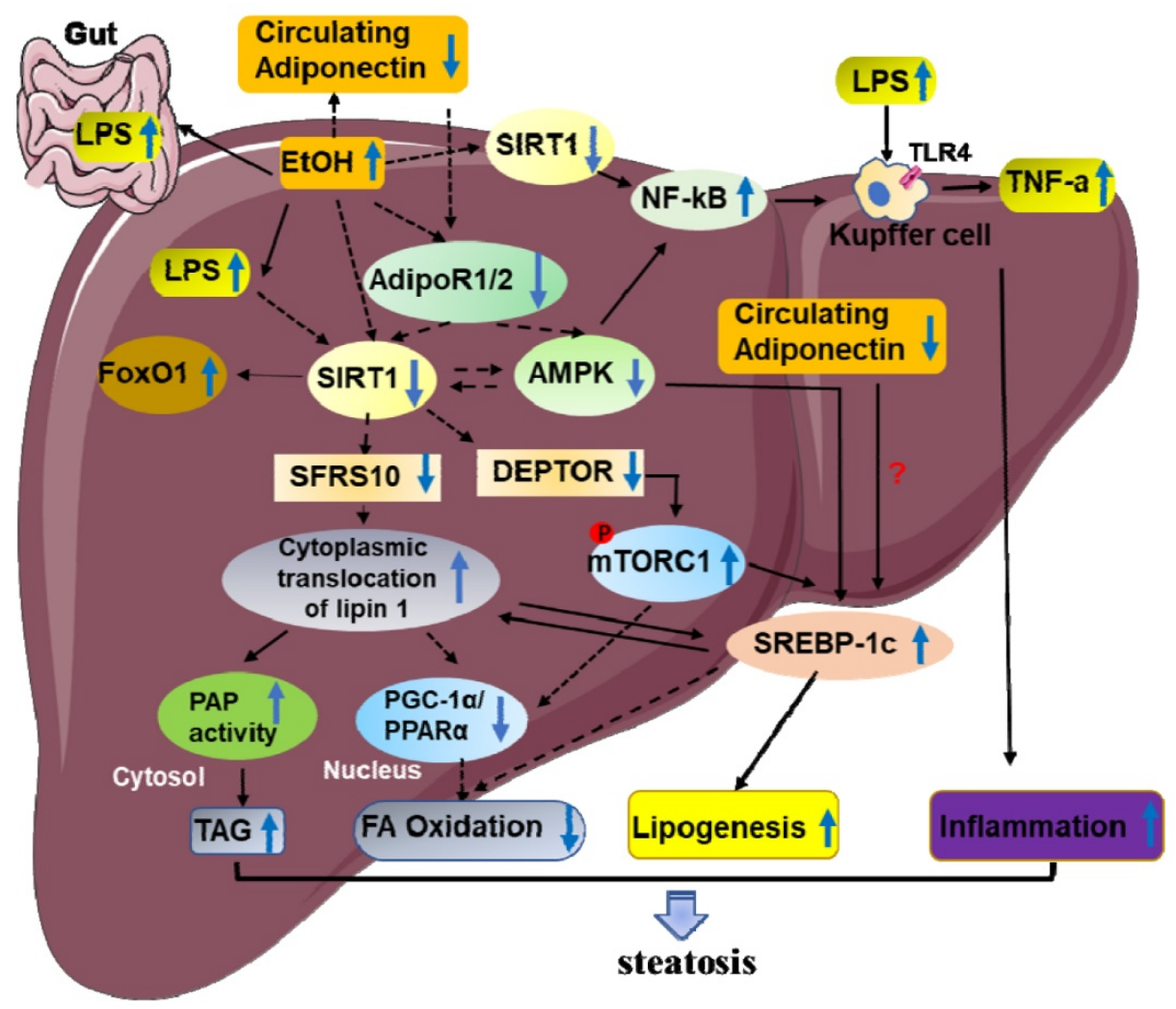

Figure 2. The roles of SIRT1 in the pathogenesis of alcoholic steatosis and inflammation. Lipogenesis and fatty acid $\beta$-oxidation are two major factors that are responsible for this impaired hepatic lipid balance in ALD. Ethanol-mediated SIRT1 inhibition and SIRT1 dysfunction promote the development of steatosis through reducing PPARa and PGC-1 $\alpha$ level, inhibiting AMPK activity, decreasing circulating adiponectin, activating PAP and mTORClactivities, elevating SREBP-1c expression. These impaired networks subsequently lead to accumulated hepatic triglycerides, increased fatty acid synthesis, decreased fatty acid oxidation, enhanced inflammatory response and steatosis development. In addition, the inhibition of SIRT1 in Kupffer cells was related to a marked increase in the acetylation of NF-KB, which leads to inflammation of ALD 
Chronic inflammation acts as a widely recognized hallmark in ageing process [80, 81]. Accumulating studies have proved that SIRT1 involved in many biological processes through regulating protein acetylation, thus, promotes longevity [82, 83]. Previous studies researched that hepatic expression of SIRT1 protein was downregulated in the middle-aged mice compared to young mice, which lead to more liver injury and inflammation induced by ethanol feeding in middle-aged mice and aged mice when compared to young [84]. These data indicate that SIRT1 is an essential negative inflammatory regulator in alcohol induced liver diseases; thereby reducing inflammatory cells infiltration and pro-inflammatory cytokines production in the liver (Figure 2).

\section{SIRTI and Alcoholic Fibrosis}

Liver fibrosis is characterized by excessive accumulation of the extracellular matrix (ECM)[85]. The cell type that is predominantly responsible for fibrotic processes is hepatic stellate cells (HSCs), which comprise approximately $5-10 \%$ of total resident cells in normal human liver [85]. It is well accepted that HSCs activation is the critical event accompanied with upregulation of transforming growth factor beta 1 (TGF- $\beta 1$ ) and activate collagen production in liver fibro-genesis [86]. Recently, the roles of SIRT1 in alcoholic fibrosis had also been widely reported [84, 87]. Studies found that overexpression of SIRT1 attenuated TGF- $\beta 1$-induced expression of myofibroblast markers [87]. Consistently, ethanol fed liver-specific deletion of SIRT1 (Sirt1LKO) promotes ethanol-mediated liver fibrosis in the livers, as indicated by increased levels of a-SMA and early markers of hepatic fibrosis such as collagen I, tissue inhibitor of metalloproteinase 1 (Timp-1), or TGF- $\beta 1$ [15]. In addition, HSC-specific SIRT1 knockout mice were more susceptible to ethanol-induced liver fibrosis with upregulation of alpha-smooth muscle actin $(a-S M A)$, platelet derived growth factor (PDGF) and Collagen alpha- $1(\mathrm{~V})$ chain (Col5a1) mRNA levels [84]. Downregulation of SIRT1 expression in HSCs from middle-aged mice contributes to the increased ethanol-induced liver injury and fibrosis, which accompanied by PDGFR-a and c-Myc expression upregulation [84]. Both PDGFR-a and c-Mycare are two of the most important factors that promotes HSC activation via TGF- $\beta$ signaling [88, 89]. Correspondingly, restoration of SIRT1expression ameliorated short-term plus binge ethanol-induced liver injury and fibrosis in middle-aged mice [84]. Therefore, activation of SIRT1 may be a potential therapeutic strategy for the treatment of ALD in elderly patients (Figure 3).

\section{SIRT1 and Hepatocellular carcinoma}

Hepatocellular carcinoma (HCC) is an increasingly diagnosed disease state in the liver for which alcohol is a leading risk factor for promoting HCC development [90]. SIRT1 is a protein deacetylase known to act as a tumor promoter or suppressor in different cancers [91-93]. Recent studies have demonstrated that SIRT1 is strongly associated with the clinical outcomes of HCC. In HCC cells, SIRT1 had a predominant nuclear localization where its expression promotes tumorigenesis, while, cytoplasmatic SIRT1 may have tumor-suppressive roles [94, 95].Although SIRT1 appears to be a promising target for preventing ALD, there are few studies that have reported the roles of SIRT1 in ethanol-fed HCC due to lack of appropriate models [96]. Previous studies demonstrated that chronic, heavy ethanol consumption accelerates hepatocellular carcinoma progression accompanied by elevated SIRT1 expression, which are strong correlated with the upregulation of PGC-1a in HCC specimen [95, 97]. Meanwhile, few literatures reported that SIRT1 was downregulated in human HCC samples and hypothesized SIRT1 functions as a potential tumor suppressor [93, 98]. These findings are somewhat paradoxical, because of many SIRT1 protective roles in ALD. Of course, some experimental and clinical evidence suggest that many unique mechanisms, like decreased immune surveillance induced by impaired NK cells function contribute to the development of HCC specifically in patients with ALD [99]. It is worthwhile to furtherly investigate whether SIRT1 regulates this pathway in alcoholic HCC (Figure 3).

\section{Conclusion \& future perspective}

ALD is a major cause of advanced liver disease worldwide. Here, we have summarized the latest progress on the roles of SIRT1 in ethanol-induced steatosis, inflammation, fibrosis, and HCC. As summarized in figure 1, ethanol exposure reduces SIRT1 gene and protein expression levels, induces SIRT1 nucleocytoplasmic shuttling. As summarized in figure 2, Ethanol-mediated impairment of hepatic SIRT1 signaling via multiple transcriptional regulators and co-regulators in the liver contributes to development of alcoholic steatosis and inflammation. As summarized in figure 3, downregulation of SIRT1 in hepatocytes and HSCs contributes to ethanol-induced liver injury and fibrosis in mice, while elevated SIRT1 expression accelerates hepatocellular carcinoma progression. Even though the mechanism of the roles of SIRT1 in alcoholic liver disease has been extensively explored, there are still many problems that need to be explored. 


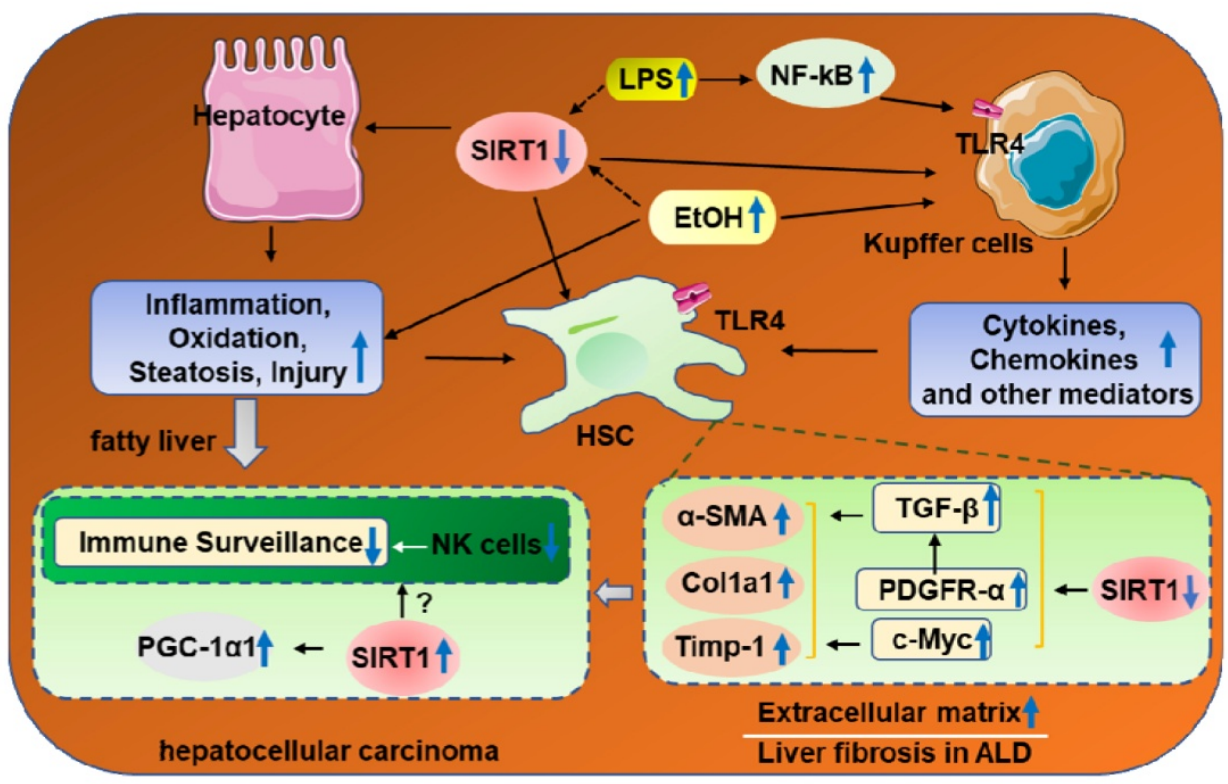

Figure 3. The roles of SIRT1 in the progression of alcoholic liver fibrosis and HCC. Chronic ethanol exposure result in elevated release of LPS, which enters the liver and activates resident macrophages (Kupffer cells), release proinflammatory cytokines and chemokines, eventually promote HSC activation via downregulation of PDGFa and c-Myc. Meanwhile, downregulation of SIRT1 in hepatocytes and HSCs contributes to the increased susceptibility to ethanol-induced liver injury and fibrosis in mice. Chronic, heavy ethanol consumption accelerates hepatocellular carcinoma progression accompanied with elevated SIRTI expression, which are strong correlated with the upregulation of PGC-1 $1 \alpha$ in HCC specimen. Interestingly, HCC generation partly due to impaired NK cell function in ALD.

Firstly, the more precise mechanisms by which ethanol inhibits SIRT1 activity will require further elucidation. Given that SIRT1 can be regulated at multiple posttranslational modifications and these epigenetic alterations can contribute to the initiation and progression of ALD, it will be important to identify whether ethanol affects acetylation, phosphorylation, O-linked $\mathrm{N}$-acetyl $\beta$-D glucosamine (O-GlcNAcylation) and the 3- untranslated region (UTR) of mRNA modification of SIRT1, which all can eventually weaken the activity of SIRT1.Previous studies have been reported IL-6 stimulation enhanced JAK1-mediated Sirt1 phosphorylation [100]. Increased O-GlcNAcylation level dynamically modifies SIRT1 at Ser-549 thus enhancing its catalytic activity when the cell is under stress condition [101]. RNA-binding protein RPS3 contributes to hepatocarcinogenesis by post-transcriptionally up-regulating SIRT1 [102]. Therefore, it will be important to determine whether and how ethanol mediated SIRT1 undergoes these post-transcriptional modifications during the ALD process.

Beside, HCC is a heterogeneous tumor associated with multiple molecules and various signaling pathways in its development and progression [103]. Although the role of SIRT1 within the progression of HCC has been intensively studied in recent years, the roles of SIRT1 in liver cancer are still controversial. In some reports, SIRT1 is frequently overexpressed in HCC, where it promotes tumorigenic, metastasis, and chemoresistance [94]. However, in other reports it was also shown that
SIRT1 protein levels were decreased in HCC when compared to their normal controls [98]. Therefore, the overexpression of SIRT1 resulted in antitumor effect in HCC [104]. The question of whether SIRT1 is a tumor suppressor or oncogene in HCC caused by long-term heavy drinking remains unclear. Furtherly, it's necessary to identify the roles of SIRT1 in different pathological stages of ethanol-induced HCC.

Lastly, an increasing number of studies suggest that natural compounds might provide a novel strategy for ALD treatment [105]. Resveratrol, SIRT1 activator, is the most extensively studied and promising for ALD[106, 107].Unfortunately, even though beneficial action of resveratrol has been well established in ALD animal models, resveratrol is restricted in clinical application by its poor oral bioavailability, chemical stability and low water solubility [37].

\section{Acknowledgements}

This study was supported in part by the National Nature and Science Function of China grant 81770588 to Hua Wang.

\section{Author Contributions}

Ruixue Ren had the idea for the article. Literature search was performed by Ziming Wang. The manuscript was written by Ruixue Ren and Hua Wang. Miaomiao Wu critically revised the work. All authors read and approved the final manuscript. 


\section{Abbreviations}

ADH: alcohol dehydrogenase; ALDH: aldehyde dehydrogenase; ROS: Reactive oxygen species; CYP2E1: Cytochrome P450 isoenzyme 2E1; NAD+: Nicotinamide adenine dinucleotide; NADH: Nicotinamide adenine dinucleotide reduced; MDA: malondialdehyde; 4-HNE: 4-hydroxy-2-nonenal; miRNAs: microRNAs; LPS: lipopolysaccharide; AdipoR: adiponectin receptor; NF-kB: nuclear transcription factor-kB; SIRT1: sirtuin 1; AMPK: AMP-activated kinase; SFRS10: serine/argininerich splicing factor 10; PAP: phosphatidic acid phosphohydrolase; PPARa: peroxisome proliferatoractivated receptor a; PGC-1a: PPARY co-activator-1a; TAGs: triglycerides; FA: fatty acid; DEPTOR: mTOR-interacting protein; mTORC1: mTOR ( mechanistic target of rapamycin) complex 1 ; SREBP-1c: sterol regulatory element-binding proteins-1c; FoxO1: Forkhead box transcription factor O1; TLR4: toll like receptor 4; HSC: Hematopoietic stem cell; NF-kB: nuclear transcription factor-kB; TLR4: toll like receptor 4 ; TGF- $\beta$ : transforming growth factor beta; PDGFR-a: PDGF receptor- $\alpha$; $\alpha$-Sma: alpha-smooth muscle actin; Timp-1: tissue inhibitor of metalloproteinase 1; NK cells: Natural killer cells; LPS: Lipopolysaccharide; PGC-1a: PPARY co-activator-1a.

\section{Competing Interests}

The authors have declared that no competing interest exists.

\section{References}

1. Younossi ZM, Stepanova M, Younossi Y, Golabi P, Mishra A, Rafiq N, et al. Epidemiology of chronic liver diseases in the USA in the past three decades. Gut. 2020; 69: 564-8.

2. Gao B, Bataller R. Alcoholic liver disease: pathogenesis and new therapeutic targets. Gastroenterology. 2011; 141: 1572-85.

3. Sharma P, Arora A. Clinical presentation of alcoholic liver disease and non-alcoholic fatty liver disease: spectrum and diagnosis. Transl Gastroenterol Hepatol. 2020; 5: 19.

4. Purohit V, Brenner DA. Mechanisms of alcohol-induced hepatic fibrosis: a summary of the Ron Thurman Symposium. Hepatology. 2006; 43: 872-8.

5. Finkel T, Deng CX, Mostoslavsky R. Recent progress in the biology and physiology of sirtuins. Nature. 2009; 460: 587-91.

6. Hall JA, Dominy JE, Lee Y, Puigserver P. The sirtuin family's role in aging and age-associated pathologies. J Clin Invest. 2013; 123: 973-9.

7. Haigis MC, Sinclair DA. Mammalian sirtuins: biological insights and disease relevance. Annu Rev Pathol. 2010; 5: 253-95.

8. Herranz D, Serrano M. SIRT1: recent lessons from mouse models. Nat Rev Cancer. 2010; 10: 819-23.

9. Ma Y, Chai H, Ding Q, Qian Q, Yan Z, Ding B, et al. Hepatic SIRT3 Upregulation in Response to Chronic Alcohol Consumption Contributes to Alcoholic Liver Disease in Mice. Front Physiol. 2019; 10: 1042.

10. Kim HG, Huang M, Xin Y, Zhang Y, Zhang X, Wang G, et al. The epigenetic regulator SIRT6 protects the liver from alcohol-induced tissue injury by reducing oxidative stress in mice. J Hepatol. 2019; 71: 960-9.

11. You M, Jogasuria A, Taylor C, Wu J. Sirtuin 1 signaling and alcoholic fatty liver disease. Hepatobiliary Surg Nutr. 2015; 4: 88-100.

12. Schug TT, Li X. Sirtuin 1 in lipid metabolism and obesity. Ann Med. 2011; 43: 198-211.

13. Chang HC, Guarente L. SIRT1 and other sirtuins in metabolism. Trends Endocrinol Metab. 2014; 25: 138-45.

14. Li Y, Wong K, Giles A, Jiang J, Lee JW, Adams AC, et al. Hepatic SIRT1 attenuates hepatic steatosis and controls energy balance in mice by inducing fibroblast growth factor 21. Gastroenterology. 2014; 146: 539-49 e7.
15. Yin $\mathrm{H}, \mathrm{Hu} \mathrm{M}$, Liang $\mathrm{X}$, Ajmo JM, Li X, Bataller $\mathrm{R}$, et al. Deletion of SIRT1 from hepatocytes in mice disrupts lipin-1 signaling and aggravates alcoholic fatty liver. Gastroenterology. 2014; 146: 801-11.

16. Nascimento AF, Ip BC, Luvizotto RA, Seitz HK, Wang XD. Aggravation of nonalcoholic steatohepatitis by moderate alcohol consumption is associated with decreased SIRT1 activity in rats. Hepatobiliary Surg Nutr. 2013; 2: 252-9.

17. Yin $\mathrm{H}, \mathrm{Hu} \mathrm{M}$, Zhang $\mathrm{R}$, Shen Z, Flatow L, You M. MicroRNA-217 promotes ethanol-induced fat accumulation in hepatocytes by down-regulating SIRT1. J Biol Chem. 2012; 287: 9817-26

18. Assadiasl S, Mooney N, Mohebbi B, Fatahi Y, Soleimanifar N. Sirtuin 1: A Dilemma in Transplantation. J Transplant. 2020; 2020: 9012980.

19. Revollo JR, Li X. The ways and means that fine tune Sirt1 activity. Trends Biochem Sci. 2013; 38: 160-7.

20. Kubiak-Tomaszewska G, Tomaszewski P, Pachecka J, Struga M, Olejarz W, Mielczarek-Puta M, et al. Molecular mechanisms of ethanol biotransformation: enzymes of oxidative and nonoxidative metabolic pathways in human. Xenobiotica. 2020; 50: 1180-201.

21. Cho YE, Kim DK, Seo W, Gao B, Yoo SH, Song BJ. Fructose Promotes Leaky Gut, Endotoxemia, and Liver Fibrosis Through Ethanol-Inducible Cytochrome P450-2E1-Mediated Oxidative and Nitrative Stress. Hepatology. 2019; [Epub ahead of print].

22. Shen Z, Ajmo JM, Rogers CQ, Liang X, Le L, Murr MM, et al. Role of SIRT1 in regulation of LPS- or two ethanol metabolites-induced TNF-alpha production in cultured macrophage cell lines. Am J Physiol Gastrointest Liver Physiol. 2009; 296: G1047-53.

23. Tanno M, Sakamoto J, Miura T, Shimamoto K, Horio Y. Nucleocytoplasmic shuttling of the NAD+-dependent histone deacetylase SIRT1. J Biol Chem. 2007; 282: 6823-32

24. Liang X, Hu M, Rogers CQ, Shen Z, You M. Role of SIRT1-FoxO1 signaling in dietary saturated fat-dependent upregulation of liver adiponectin receptor 2 in ethanol-administered mice. Antioxid Redox Signal. 2011; 15: 425-35.

25. Lieber CS, Leo MA, Wang X, Decarli LM. Effect of chronic alcohol consumption on Hepatic SIRT1 and PGC-1alpha in rats. Biochem Biophys Res Commun. 2008; 370: 44-8

26. You M, Liang X, Ajmo JM, Ness GC. Involvement of mammalian sirtuin 1 in the action of ethanol in the liver. Am J Physiol Gastrointest Liver Physiol. 2008; 294: G892-8.

27. Yin $H$, Liang $X$, Jogasuria $A$, Davidson NO, You $M$, miR-217 regulates ethanol-induced hepatic inflammation by disrupting sirtuin 1-lipin-1 signaling. Am J Pathol. 2015; 185: 1286-96.

28. Meng F, Glaser SS, Francis H, Yang F, Han Y, Stokes A, et al. Epigenetic regulation of miR-34a expression in alcoholic liver injury. Am J Pathol. 2012; 181: 804-17.

29. Choi SE, Kemper JK. Regulation of SIRT1 by microRNAs. Mol Cells. 2013; 36: 385-92.

30. D'Adamo S, Cetrullo S, Guidotti S, Borzi RM, Flamigni F. Hydroxytyrosol modulates the levels of microRNA-9 and its target sirtuin-1 thereby counteracting oxidative stress-induced chondrocyte death. Osteoarthritis Cartilage. 2017; 25: 600-10.

31. Ding S, Liu D, Wang L, Wang G, Zhu Y. Inhibiting MicroRNA-29a Protects Myocardial Ischemia-Reperfusion Injury by Targeting SIRT1 and Suppressing Oxidative Stress and NLRP3-Mediated Pyroptosis Pathway. J Pharmacol Exp Ther. 2020; 372: 128-35

32. Zhang C, Fang L, Liu X, Nie T, Li R, Cui L, et al. miR-22 inhibits synovial fibroblasts proliferation and proinflammatory cytokine production in RASF via targeting SIRT1. Gene. 2020; 724: 144144

33. Wu L, Zhang G, Guo C, Zhao X, Shen D, Yang N. MiR-128-3p mediates TNF-alpha-induced inflammatory responses by regulating Sirt1 expression in bone marrow mesenchymal stem cells. Biochem Biophys Res Commun. 2020; 521: 98-105.

34. Tobita T, Guzman-Lepe J, Takeishi K, Nakao T, Wang Y, Meng F, et al. SIRT1 Disruption in Human Fetal Hepatocytes Leads to Increased Accumulation of Glucose and Lipids. PLoS One. 2016; 11: e0149344.

35. Ajmo JM, Liang X, Rogers CQ, Pennock B, You M. Resveratrol alleviates alcoholic fatty liver in mice. Am J Physiol Gastrointest Liver Physiol. 2008; 295: G833-42.

36. de Gregorio E, Colell A, Morales A, Mari M. Relevance of SIRT1-NF-kappaB Axis as Therapeutic Target to Ameliorate Inflammation in Liver Disease. Int J Mol Sci. 2020; 21.

37. Ding RB, Bao J, Deng CX. Emerging roles of SIRT1 in fatty liver diseases. Int J Biol Sci. 2017; 13: 852-67.

38. Osborne TF. Sterol Regulatory Element-binding Proteins (SREBPs): Key Regulators of Nutritional Homeostasis and Insulin Action. Journal of Biological Chemistry. 2000; 275: 32379-82.

39. Ji C, Chan C, Kaplowitz N. Predominant role of sterol response element binding proteins (SREBP) lipogenic pathways in hepatic steatosis in the murine intragastric ethanol feeding model. J Hepatol. 2006; 45: 717-24.

40. Ponugoti B, Kim DH, Xiao Z, Smith Z, Miao J, Zang M, et al. SIRT1 deacetylates and inhibits SREBP-1C activity in regulation of hepatic lipid metabolism. J Biol Chem. 2010; 285: 33959-70.

41. You M, Matsumoto M, Pacold CM, Cho WK, Crabb DW. The role of AMP-activated protein kinase in the action of ethanol in the liver. Gastroenterology. 2004; 127: 1798-808.

42. Saxton RA, Sabatini DM. mTOR Signaling in Growth, Metabolism, and Disease. Cell. 2017; 168: 960-76. 
43. Laplante M, Sabatini DM. mTOR signaling in growth control and disease. Cell. 2012; 149: 274-93.

44. Peterson TR, Laplante M, Thoreen CC, Sancak Y, Kang SA, Kuehl WM, et al. DEPTOR is an mTOR inhibitor frequently overexpressed in multiple myeloma cells and required for their survival. Cell. 2009; 137: 873-86.

45. Gao D, Inuzuka H, Tan MK, Fukushima H, Locasale JW, Liu P, et al. mTOR drives its own activation via $\mathrm{SCF}($ betaTrCP)-dependent degradation of the mTOR inhibitor DEPTOR. Mol Cell. 2011; 44: 290-303.

46. Chen H, Shen F, Sherban A, Nocon A, Li Y, Wang H, et al. DEP domain-containing mTOR-interacting protein suppresses lipogenesis and ameliorates hepatic steatosis and acute-on-chronic liver injury in alcoholic liver disease. Hepatology. 2018; 68: 496-514.

47. Wagner M, Zollner G, Trauner M. Nuclear receptors in liver disease. Hepatology. 2011; 53: 1023-34.

48. Takada I, Makishima M. Peroxisome proliferator-activated receptor agonists and antagonists: a patent review (2014-present). Expert Opin Ther Pat. 2020; 30: 1-13.

49. Wang Y, Nakajima T, Gonzalez FJ, Tanaka N. PPARs as Metabolic Regulators in the Liver: Lessons from Liver-Specific PPAR-Null Mice. Int J Mol Sci. 2020; 21.

50. Lu Y, Zhuge J, Wang X, Bai J, Cederbaum AI. Cytochrome P450 2E1 contributes to ethanol-induced fatty liver in mice. Hepatology. 2008; 47: 1483-94.

51. Nemoto S, Fergusson MM, Finkel T. SIRT1 functionally interacts with the metabolic regulator and transcriptional coactivator PGC-1\{alpha\}. J Biol Chem. 2005; 280: 16456-60.

52. Hu M, Yin H, Mitra MS, Liang X, Ajmo JM, Nadra K, et al. Hepatic-specific lipin-1 deficiency exacerbates experimental alcohol-induced steatohepatitis in mice. Hepatology. 2013; 58: 1953-63.

53. Purushotham A, Schug TT, Xu Q, Surapureddi S, Guo X, Li X. Hepatocyte-specific deletion of SIRT1 alters fatty acid metabolism and results in hepatic steatosis and inflammation. Cell Metab. 2009; 9: 327-38.

54. $\mathrm{Li} \mathrm{C}, \mathrm{Hu} \mathrm{WL}, \mathrm{Lu} \mathrm{MX}$, Xiao GF. Resveratrol induces apoptosis of benign prostatic hyperplasia epithelial cell line (BPH-1) through p38 MAPK-FOXO3a pathway. BMC Complement Altern Med. 2019; 19: 233.

55. Nagappan A, Kim JH, Jung DY, Jung MH. Cryptotanshinone from the Salvia miltiorrhiza Bunge Attenuates Ethanol-Induced Liver Injury by Activation of AMPK/SIRT1 and Nrf2 Signaling Pathways. Int J Mol Sci. 2019; 21.

56. Rogers CQ, Ajmo JM, You M. Adiponectin and alcoholic fatty liver disease. IUBMB Life. 2008; 60: 790-7.

57. Jiang Z, Zhou J, Zhou D, Zhu Z, Sun L, Nanji AA. The adiponectin-SIRT1-AMPK pathway in alcoholic fatty liver disease in the rat. Alcohol Clin Exp Res. 2015; 39: 424-33.

58. Xu J, Lai KKY, Verlinsky A, Lugea A, French SW, Cooper MP, et al. Synergistic steatohepatitis by moderate obesity and alcohol in mice despite increased adiponectin and p-AMPK. J Hepatol. 2011; 55: 673-82.

59. Tomita K, Tamiya G, Ando S, Kitamura N, Koizumi H, Kato S, et al. AICAR, an AMPK activator, has protective effects on alcohol-induced fatty liver in rats. Alcohol Clin Exp Res. 2005; 29: 240S-5S.

60. Price NL, Gomes AP, Ling AJ, Duarte FV, Martin-Montalvo A, North BJ, et al. SIRT1 is required for AMPK activation and the beneficial effects of resveratrol on mitochondrial function. Cell Metab. 2012; 15: 675-90.

61. Zang M, Xu S, Maitland-Toolan KA, Zuccollo A, Hou X, Jiang B, et al. Polyphenols stimulate AMP-activated protein kinase, lower lipids, and inhibit accelerated atherosclerosis in diabetic LDL receptor-deficient mice. Diabetes. 2006; 55: 2180-91.

62. Harris TE, Finck BN. Dual function lipin proteins and glycerolipid metabolism. Trends Endocrinol Metab. 2011; 22: 226-33.

63. Hu M, Wang F, Li X, Rogers CQ, Liang X, Finck BN, et al. Regulation of hepatic lipin-1 by ethanol: role of AMP-activated protein kinase/sterol regulatory element-binding protein 1 signaling in mice. Hepatology. 2012; 55: 437-46.

64. Finck BN, Gropler MC, Chen Z, Leone TC, Croce MA, Harris TE, et al. Lipin 1 is an inducible amplifier of the hepatic PGC-1alpha/PPARalpha regulatory pathway. Cell Metab. 2006; 4: 199-210.

65. Peterson TR, Sengupta SS, Harris TE, Carmack AE, Kang SA, Balderas E, et al. mTOR complex 1 regulates lipin 1 localization to control the SREBP pathway. Cell. 2011; 146: 408-20.

66. You M, Jogasuria A, Lee K, Wu J, Zhang Y, Lee YK, et al. Signal Transduction Mechanisms of Alcoholic Fatty Liver Disease: Emer ging Role of Lipin-1. Curr Mol Pharmacol. 2017; 10: 226-36.

67. Maeda N, Funahashi T, Matsuzawa Y, Shimomura I. Adiponectin, a unique adipocyte-derived factor beyond hormones. Atherosclerosis. 2020; 292: 1-9.

68. Correnti JM, Juskeviciute E, Swarup A, Hoek JB. Pharmacological ceramide reduction alleviates alcohol-induced steatosis and hepatomegaly in adiponectin knockout mice. American Journal of Physiology-Gastrointestinal and Liver Physiology. 2014; 306: G959-G73.

69. Kadowaki T, Yamauchi T, Kubota N, Hara K, Ueki K, Tobe K. Adiponectin and adiponectin receptors in insulin resistance, diabetes, and the metabolic syndrome. J Clin Invest. 2006; 116: 1784-92.

70. Shen Z, Liang X, Rogers CQ, Rideout D, You M. Involvement of adiponectin-SIRT1-AMPK signaling in the protective action of rosiglitazone against alcoholic fatty liver in mice. Am J Physiol Gastrointest Liver Physiol. 2010; 298: G364-74.
71. You M, Rogers CQ. Adiponectin: a key adipokine in alcoholic fatty liver. Exp Biol Med (Maywood). 2009; 234: 850-9.

72. Song Z, Zhou Z, Deaciuc I, Chen T, McClain CJ. Inhibition of adiponectin production by homocysteine: a potential mechanism for alcoholic liver disease. Hepatology. 2008; 47: 867-79.

73. Frescas D, Valenti L, Accili D. Nuclear trapping of the forkhead transcription factor FoxO1 via Sirt-dependent deacetylation promotes expression of glucogenetic genes. J Biol Chem. 2005; 280: 20589-95.

74. Tilg H, Zmora N, Adolph TE, Elinav E. The intestinal microbiota fuelling metabolic inflammation. Nature Reviews Immunology. 2019; 20: 40-54.

75. Gao B, Ahmad MF, Nagy LE, Tsukamoto H. Inflammatory pathways in alcoholic steatohepatitis. J Hepatol. 2019; 70: 249-59.

76. Dorrington MG, Fraser IDC. NF-kappaB Signaling in Macrophages: Dynamics, Crosstalk, and Signal Integration. Front Immunol. 2019; 10: 705.

77. Kauppinen A, Suuronen T, Ojala J, Kaarniranta K, Salminen A. Antagonistic crosstalk between NF-kappaB and SIRT1 in the regulation of inflammation and metabolic disorders. Cell Signal. 2013; 25: 1939-48.

78. Schug TT, Xu Q, Gao H, Peres-da-Silva A, Draper DW, Fessler MB, et al. Myeloid deletion of SIRT1 induces inflammatory signaling in response to environmental stress. Mol Cell Biol. 2010; 30: 4712-21.

79. Pfluger PT, Herranz D, Velasco-Miguel S, Serrano M, Tschop MH. Sirt1 protects against high-fat diet-induced metabolic damage. Proc Natl Acad Sci U S A. 2008; 105: 9793-8.

80. Sendama W. The effect of ageing on the resolution of inflammation. Ageing Res Rev. 2020; 57: 101000

81. Canadas-Lozano D, Marin-Aguilar F, Castejon-Vega B, Ryffel B, Navarro-Pando JM, Ruiz-Cabello J, et al. Blockade of the NLRP3 inflammasome improves metabolic health and lifespan in obese mice. Geroscience. 2020; 42: 715-25.

82. Wang RH, Zhao T, Cui K, Hu G, Chen Q, Chen W, et al. Negative reciprocal regulation between Sirt1 and Per2 modulates the circadian clock and aging. Sci Rep. 2016; 6: 28633.

83. Ishizuka K, Kon K, Lee-Okada HC, Arai K, Uchiyama A, Yamashina S, et al. Aging exacerbates high-fat diet-induced steatohepatitis through alteration in hepatic lipid metabolism in mice. J Gastroenterol Hepatol. 2020; 35: 1437-48.

84. Ramirez T, Li YM, Yin S, Xu MJ, Feng D, Zhou Z, et al. Aging aggravates alcoholic liver injury and fibrosis in mice by downregulating sirtuin 1 expression. J Hepatol. 2017; 66: 601-9.

85. Higashi T, Friedman SL, Hoshida Y. Hepatic stellate cells as key target in liver fibrosis. Adv Drug Deliv Rev. 2017; 121: 27-42.

86. Bataller R, Brenner DA. Liver fibrosis. Journal of Clinical Investigation. 2005; 115: 209-18.

87. Wu Y, Liu X, Zhou Q, Huang C, Meng X, Xu F, et al. Silent information regulator 1 (SIRT1) ameliorates liver fibrosis via promoting activated stellate cell apoptosis and reversion. Toxicol Appl Pharmacol. 2015; 289: 163-76.

88. Czochra P, Klopcic B, Meyer E, Herkel J, Garcia-Lazaro JF, Thieringer F, et al. Liver fibrosis induced by hepatic overexpression of PDGF-B in transgenic mice. J Hepatol. 2006; 45: 419-28.

89. Arteaga M, Shang N, Ding X, Yong S, Cotler SJ, Denning MF, et al. Inhibition of SIRT2 suppresses hepatic fibrosis. Am J Physiol Gastrointest Liver Physiol. 2016; 310: G1155-68.

90. Mendez-Sanchez N, Valencia-Rodriguez A, Vera-Barajas A, Abenavoli L, Scarpellini E, Ponciano-Rodriguez G, et al. The mechanism of dysbiosis in alcoholic liver disease leading to liver cancer. Hepatoma Res. 2020; 6:5

91. Chen J, Zhang B, Wong N, Lo AW, To KF, Chan AW, et al. Sirtuin 1 is upregulated in a subset of hepatocellular carcinomas where it is essential for telomere maintenance and tumor cell growth. Cancer Res. 2011; 71: 4138-49.

92. Liu T, Liu PY, Marshall GM. The critical role of the class III histone deacetylase SIRT1 in cancer. Cancer Res. 2009; 69: 1702-5.

93. Fang $Y$, Nicholl MB. Sirtuin 1 in malignant transformation: friend or foe? Cancer Lett. 2011; 306: 10-4.

94. Farcas M, Gavrea AA, Gulei D, Ionescu C, Irimie A, Catana CS, et al. SIRT1 in the Development and Treatment of Hepatocellular Carcinoma. Front Nutr. 2019; 6: 148.

95. Lin XL, Li K, Yang Z, Chen B, Zhang T. Dulcitol suppresses proliferation and migration of hepatocellular carcinoma via regulating SIRT1/p53 pathway. Phytomedicine. 2020; 66: 153112.

96. Mathews S, Xu M, Wang $\mathrm{H}$, Bertola A, Gao B. Animals models of gastrointestinal and liver diseases. Animal models of alcohol-induced liver disease: pathophysiology, translational relevance, and challenges. Am J Physiol Gastrointest Liver Physiol. 2014; 306: G819-23.

97. Brandon-Warner E, Walling TL, Schrum LW, McKillop IH. Chronic ethanol feeding accelerates hepatocellular carcinoma progression in a sex-dependent manner in a mouse model of hepatocarcinogenesis. Alcohol Clin Exp Res. 2012; 36: 641-53.

98. Wang RH, Sengupta K, Li C, Kim HS, Cao L, Xiao C, et al. Impaired DNA damage response, genome instability, and tumorigenesis in SIRT1 mutant mice. Cancer Cell. 2008; 14: 312-23.

99. Morgan TR, Mandayam S, Jamal MM. Alcohol and hepatocellular carcinoma. Gastroenterology. 2004; 127: S87-96.

100. Wang W, Li F, Xu Y, Wei J, Zhang Y, Yang H, et al. JAK1-mediated Sirt1 phosphorylation functions as a negative feedback of the JAK1-STAT3 pathway. J Biol Chem. 2018; 293: 11067-75. 
101. Han C, Gu Y, Shan H, Mi W, Sun J, Shi M, et al. O-GlcNAcylation of SIRT1 enhances its deacetylase activity and promotes cytoprotection under stress. Nat Commun. 2017; 8: 1491.

102. Zhao L, Cao J, Hu K, Wang P, Li G, He X, et al. RNA-binding protein RPS3 contributes to hepatocarcinogenesis by post-transcriptionally up-regulating SIRT1. Nucleic Acids Res. 2019; 47: 2011-28.

103. Xue R, Li R, Guo H, Guo L, Su Z, Ni X, et al. Variable Intra-Tumor Genomic Heterogeneity of Multiple Lesions in Patients With Hepatocellular Carcinoma. Gastroenterology. 2016; 150: 998-1008.

104. Zhang XL, Yu H, Xiong YY, Ma ST, Zhao L, She SF. Resveratrol down-regulates Myosin light chain kinase, induces apoptosis and inhibits diethylnitrosamine-induced liver tumorigenesis in rats. Int J Mol Sci. 2013; 14: 1940-51.

105. Tu Y, Zhu S, Wang J, Burstein E, Jia D. Natural compounds in the chemoprevention of alcoholic liver disease. Phytother Res. 2019; 33: 2192-212.

106. Luo G, Xiao L, Wang D, Wang N, Luo C, Yang X, et al. Resveratrol protects against ethanol-induced impairment of insulin secretion in INS-1 cells through SIRT1-UCP2 axis. Toxicol In Vitro. 2020; 65: 104808.

107. Szkudelski T, Szkudelska K. Potential of resveratrol in mitigating metabolic disturbances induced by ethanol. Biomed Pharmacother. 2018; 101: 579-84. 